\title{
The Effect of Interpersonal Psychotherapy and other Psychodynamic Therapies versus 'Treatment as Usual' in Patients with Major Depressive Disorder
}

\author{
Janus Christian Jakobsen ${ }^{1,2 *}$, Jane Lindschou Hansen ${ }^{1}$, Erik Simonsen ${ }^{1,2}$, Christian Gluud ${ }^{1}$ \\ 1 Copenhagen Trial Unit, Centre for Clinical Intervention Research, Department 3344 Rigshospitalet, Copenhagen University Hospital, Copenhagen, Denmark, 2 Psychiatric \\ Research Unit, Copenhagen University Hospital and Region Zealand, Roskilde, Denmark
}

\begin{abstract}
Background: Major depressive disorder afflicts an estimated $17 \%$ of individuals during their lifetimes at tremendous suffering and costs. Interpersonal psychotherapy and other psychodynamic therapies may be effective interventions for major depressive disorder, but the effects have only had limited assessment in systematic reviews.

Methods/Principal Findings: Cochrane systematic review methodology with meta-analysis and trial sequential analysis of randomized trials comparing the effect of psychodynamic therapies versus 'treatment as usual' for major depressive disorder. To be included the participants had to be older than 17 years with a primary diagnosis of major depressive disorder. Altogether, we included six trials randomizing a total of 648 participants. Five trials assessed 'interpersonal psychotherapy' and only one trial assessed 'psychodynamic psychotherapy'. All six trials had high risk of bias. Meta-analysis on all six trials showed that the psychodynamic interventions significantly reduced depressive symptoms on the 17-item Hamilton Rating Scale for Depression (mean difference -3.12 (95\% confidence interval -4.39 to $-1.86 ; \mathrm{P}<0.00001$ ), no heterogeneity) compared with 'treatment as usual'. Trial sequential analysis confirmed this result.
\end{abstract}

Discussion: We did not find convincing evidence supporting or refuting the effect of interpersonal psychotherapy or psychodynamic therapy compared with 'treatment as usual' for patients with major depressive disorder. The potential beneficial effect seems small and effects on major outcomes are unknown. Randomized trials with low risk of systematic errors and low risk of random errors are needed.

Citation: Jakobsen J, Hansen JL, Simonsen E, Gluud C (2011) The Effect of Interpersonal Psychotherapy and other Psychodynamic Therapies versus 'Treatment as Usual' in Patients with Major Depressive Disorder. PLoS ONE 6(4): e19044. doi:10.1371/journal.pone.0019044

Editor: Isabelle Boutron, University Paris Descartes, France

Received January 23, 2011; Accepted March 25, 2011; Published April 27, 2011

Copyright: (C) 2011 Jakobsen et al. This is an open-access article distributed under the terms of the Creative Commons Attribution License, which permits unrestricted use, distribution, and reproduction in any medium, provided the original author and source are credited.

Funding: The authors have no support or funding to report

Competing Interests: Dr. Christian Gluud is a PLoS ONE Academic Editor. The authors declare that no other competing interests exist.

*E-mail: janusjakobsen@mac.com

\section{Introduction}

According to the WHO, major depressive disorder is the second largest healthcare problem worldwide in terms of illness induced disability [1]. Major depressive disorder afflicts an estimated $17 \%$ of individuals during their lifetimes at tremendous costs to the individual and society [2,3], and roughly a third of all depressive disorders take a chronic course [4,5]. Compared to other medical disorders, depressive illness causes the most significant deterioration in individual life quality [6]. Approximately $15 \%$ of depressive patients will commit suicide over a 10-20 year period [7].

Antidepressant medication remains the mainstay in the treatment of depression [8]. However, meta-analyses have shown that the new antidepressants only obtained beneficial effect in severely depressed patients and that this effect was clinically small $[9,10]$. Antidepressants are, however, known to decrease the risk of relapse [11]. The benefits of antidepressant medication seem to be limited and this raises the question if there are other effective treatments for this serious illness?

Psychodynamic therapies origin back to Freud [12]. In some health-care systems it is currently the most commonly used form of psychotherapy [13]. Interpersonal psychotherapy is generally considered as one of the most evidence-based therapies for depression [13]. Interpersonal psychotherapy originates from classical psychodynamic therapy [14], and although interpersonal psychotherapy has integrated elements from other psychotherapies it is generally regarded as a contemporary form of psychodynamic therapy $[14,15]$. We have only been able to identify one relevant meta-analysis examining the effects of psychodynamic therapies versus 'treatment as usual' for major depressive disorder [16]. The authors found that psychodynamic therapy is more effective than 'treatment as usual' for depression [16]. However, the metaanalysis did not include thorough assessment of bias risk in the included trials, did not include trials using interpersonal psychotherapy as experimental intervention, and did not employ trial sequential analysis or other methods to reduce the risk of random errors [17-19]. We therefore embarked on a systematic review using Cochrane methodology to assess the effect of interpersonal psychotherapy and other psychodynamic therapies versus 'treatment as usual' [20]. We used assessment of bias risk to reduce systematic errors [20], and trial sequential analysis to reduce the risk of random errors [17-19]. 


\section{Methods}

We conducted our systematic review of randomized clinical trials involving meta-analysis [20] and trial sequential analysis $[18,19,21]$ to answer the question: what are the beneficial and harmful effects of psychodynamic therapies versus 'treatment as usual' in the treatment of major depressive disorder?

For details regarding the methodology please consult our protocol published on our website (www.ctu.dk) in February 2010 before we began data extraction and analysis [22].

In short, we included all randomized clinical trials comparing the effects of interpersonal psychotherapy or other psychodynamic therapies versus 'treatment as usual' - irrespective of language, publication status, publication year, and publication type based on searches in The Cochrane Library's CENTRAL, MEDLINE via PubMed, EMBASE, Psychlit, Psyc Info, and Science Citation Index Expanded. The timeframe for the search was all trials published before February 2010.

To be included participants had to be older than 17 years with a primary diagnosis of major depressive disorder. Trials were only included if the diagnosis of depression was based on one of the standardized criteria, such as DSM IV [23], ICD 10 [24], DSM III [25], or DSM III-R [26].

Co-morbidity with other psychiatric diagnoses was not an exclusion criterion. The following types of trials were excluded:

- Trials focusing on depressed participants with co-morbid serious somatic illness, e.g., myocardial infarction, multiple sclerosis, cerebral stroke, cancer, etc.

- Trials focusing on 'late life' depression or depression in the elderly, most often participants over 65 years.

- Trials focusing on pregnancy-related depression, e.g., postpartum depression, postnatal depression, etc.

- Drug or alcohol dependence-related depression.

These exclusions were conducted because we expect participants in such trials to respond differently to standardized psychotherapy than other depressed patients, and these types of depressed patients are traditionally examined in separate trials [27-30].

\section{Interventions}

To be included the trials had to use at least one of the following interventions:

- Trials using interpersonal psychotherapy $[14,15]$.

- Psychotherapeutic methods based on one of the classic developers of psychodynamic therapies such as Sifneos, Malan, Mann, Davanloo, or Luborsky [31].

- The notions of transference and counter-transference (raising awareness of the therapeutic relationship) [32].

Furthermore, the trials had to present a treatment manual and had to document adherence to the treatment manual for the interventions to be classified as 'adequately defined'. All other trials that used interventions classified as 'interpersonal', 'psychodynamic', or 'dynamic' were included, but the interventions were classified under 'not adequately defined'.

For 'treatment as usual' control interventions we accepted any non-specific treatments described as: 'treatment as usual', 'standard care', or 'clinical management'. To be included the 'treatment as usual' condition had to include some kind of nonspecific supportive treatment.
Trials comparing psychodynamic therapies versus 'treatment as usual' as add-on therapy to any co-intervention were included only if these co-interventions were described and administered similarly to the different intervention groups.

Two of the review authors (JJ and JLH) independently selected relevant trials. If a trial only was identified by one of the two, it was discussed whether the trial should be included. Excluded trials were entered on a list, stating the reason for exclusion.

\section{Data extraction}

Data were extracted for trial design, bias risk, and outcomes independently by two authors (JJ and JLH). Disagreements were resolved by discussion or through arbitration $(\mathrm{CG})$. We used the instructions in The Cochrane Handbook for Systematic Reviews of Interventions [20] in our evaluation of the methodology and hence bias risk of the included trials. We assessed the bias risk in respect to generation of the allocation sequence, allocation concealment, blinding, intention-to-treat analysis, drop-outs, reporting of outcome measures, economic bias, and academic bias. These components enable classification of the included trials into trials with 'low risk of bias' or with 'high risk of bias'. The trials were overall classified as 'high risk of bias' if one or more of the above components was 'uncertain' or 'high risk of bias' [20,3336]. This classification is important because trials with 'high risk of bias' may overestimate positive intervention effects and underestimate negative effects $[20,33,34,36]$, and we wanted to relate the validity of our results to the risk of bias in the included trials.

\section{Primary outcome measures}

Depressive symptoms. Our primary outcome was the mean value of the Hamilton Rating Scale for Depression (HDRS) [37], Becks Depression Inventory (BDI) [38], or Montgomery-Asberg Depression Rating Scale (MADRS) [39] at follow-up. We included data based on the total number of randomized patients (intention-to-treat analysis) if these data were reported. We planned to estimate the therapeutic follow-up responses at two time points:

- At cessation of treatment: The trials original primary choice of completion date was used. This was the most important outcome measure time point in this review.

- At maximum follow-up.

Adverse events. We classified adverse events as serious or non-serious. Serious adverse events were defined as medical events that are life threatening; result in death; disability or significant loss of function; that cause hospital admission or prolonged hospitalization; a hereditary anomaly; or fetal injury [40]. All other adverse events (that is, events that have not necessarily had a causal relationship with the treatment, but that resulted in a change in- or cessation of the treatment) were considered as nonserious events.

Quality of life. We included any measure of quality of life, noting each assessment measure.

\section{Secondary outcome measures}

Participants without remission. The proportion of participants not having achieved remission. We included data based on the total number of randomized participants (intentionto-treat analysis) - if at all possible. If the results were not based on the total number of participants, we preformed an intention-totreat analysis assuming that the participants not included in the results did not achieve remission [20]. We pragmatically defined 
remission as HDRS of less than 8, BDI less than 10, or MADRS less than 10 [37-39].

Participants with suicidal inclination. Number of suicide inclination, suicide attempts, or suicides.

\section{Statistical methods}

This meta-analysis was undertaken according to the recommendations stated in The Cochrane Handbook for Systematic Reviews of Interventions [20]. In analyzing continuous outcomes with both fixed-effect and with random-effects models, we used the mean difference $(\mathrm{MD})$ with a $95 \%$ confidence interval. We used RevMan version 5.0 [41]. We did not use 'standardized mean difference' so each outcome measure was analyzed separately. We did not adjust the outcome variables at follow-up according to the baseline values [20].

We used the odds ratio with a $95 \%$ confidence interval to estimate intervention effects on dichotomous outcomes with both fixed-effect and with random-effects models [41].

For primary outcome measures, we also conducted trial sequential analyses. In order to calculate the required information size and the cumulative Z-curve's eventual breach of relevant trial sequential monitoring boundaries $[17,18]$, the trial sequential analysis was based on a type I error of $5 \%$, a beta of $10 \%$ (power of $90 \%$ ), the variance of all the trials (as no trial had low risk of bias), and a minimal relevant difference of 2 points on the HDRS.

\section{Results}

\section{Search results}

Our primary literature search identified 3212 publications. According to our protocol [22] we excluded 3170 publications either because they did not relate to psychodynamic therapies and major depressive disorder, or because they were not randomized trials comparing psychodynamic therapies versus 'treatment as usual'. 2831 of the 3170 were excluded on the basis of the title or abstract and 339 of the 3170 were excluded on the basis of the full publication.

Further 25 publications [42-66] were excluded because the trial participants or the interventions did not meet our inclusion criteria.

Included trials. We identified 17 publications [56-58,6780] on six trials $[56,67,69,75,79,80]$, randomizing a total of 648 participants (Figure S1). The experimental interventions were by the trialists classified as 'interpersonal psychotherapy' in five trials $[56,69,75,79,80]$ and as 'psychodynamic psychotherapy' in one trial [67].

Only three of the trials $[67,75,80]$ used an intervention that we classified as 'adequately defined'. We classified the therapists' level of experience and/or education one trial as 'high' [75], in two trials as 'intermediate' $[67,69]$, and in the last three as 'unclear' $[56,79,80]$. One trial used a combination of group therapy and individual therapy [69], the remaining five used only individual therapy $[56,67,75,79,80]$.

The duration and the extend of the psychotherapy varied in the different trials from five weeks of treatment [69] to 16 weekly sessions followed by four monthly sessions [79].

The form and extend of the 'treatment as usual' interventions varied greatly between all of the included trials (Table 1).

Two trials used antidepressants in both intervention groups $[67,69]$. Burnand et al. used clomipramine [67] and Schramm et al. used sertralin and amitriptyline [69]. The antidepressant medicine was delivered similarly in the experimental and control groups in both trials.
DiMascio et al. examined the effect of interpersonal psychotherapy versus 'non-scheduled treatment' [56]. The participants were assessed with HDRS and The Raskin Depression Scale [81]. The results at end of treatment show a significant effect of Interpersonal psychotherapy compared to 'non- scheduled treatment', but no significant difference was found at one-year followup. However, the trial did not report the SD for the mean values. We have written to the authors requesting the necessary data - but we have received no answer. Therefore, we have not been able to include the results from this trial in the following analysis.

Table 1 summarizes the characteristics of the six included trials.

Bias risk. We assessed all six trials $[56,67,69,75,79,80]$ as having 'high risk of bias' due to unclear or inadequate components as described in table 2.

\section{Effects of psychodynamic therapy}

Primary outcome measures. Five trials assessed HDRS as a continuous outcome measure at the end of treatment $[67,69,75,79,80]$. Three trials also assessed BDI $[69,75,80]$.

HDRS. Meta-analysis with fixed-effect model on the HDRS data from the five trials $[67,69,75,79,80]$ shows that psychodynamic therapies at cessation of treatment significantly reduced depressive symptoms compared with 'treatment as usual'. We found a mean difference on -3.12 HDRS $(95 \%$ CI -4.39 to $\left.-1.86 ; \quad \mathrm{P}<0.00001, \mathrm{I}^{2}=0\right)$. The $\mathrm{I}^{2}$ statistic describes the percentage of variation across trials that are due to heterogeneity rather than chance. Sub-analysis with fixed-effect model on the HDRS-data from the four trials assessing interpersonal psychotherapy $[69,75,79,80]$ also showed a similar reduction compared with 'treatment as usual' $(\mathrm{P}<0.00001)$. However, the results from the one trial assessing psychodynamic psychotherapy [82] did not show any significant difference in effect $(\mathrm{P}=0.63)$ (Figure 1).

Two of the trials reported assessment data on the HDRS after the cessation of treatment $[69,80]$. Schramm et al. assessed the participants at 12 months after cessation of treatment [69]. Swartz et al. assessed at nine months after the beginning of treatment [80]. Meta-analysis with fixed-effect model on these data showed a mean difference on -4.61 HDRS $(95 \%$ CI -6.98 to -2.24 ; $\left.\mathrm{P}<0.0001, \mathrm{I}^{2}=0\right)$ in favor of psychodynamic therapies. Both trials assessed interpersonal psychotherapy.

We performed a 'test of interaction' [83] to analyze if the effect of two kinds of psychodynamic therapy differed between the three trials assessing 'interpersonal psychotherapy' $[69,75,79,00]$ and the one trial assessing 'short psychodynamic supportive psychotherapy' [67]. 'Test of interaction' showed no significant difference $(\mathrm{P}=0.13)$, indicating that the effects of these two types of psychodynamic therapy do not seem to differ.

Trial sequential analysis on the HDRS-data also showed a significant beneficial effect of psychodynamic therapy compared with 'treatment as usual' (figure 2).

BDI. Meta-analysis with fixed-effect model on the data from the three trials $[69,75,80]$ reporting results on the BDI at cessation of treatment were in agreement with the results from the HDRS (mean difference on -3.09 BDI $(95 \%$ CI -5.35 to -0.83 ; $\left.\mathrm{P}=0.007, \quad \mathrm{I}^{2}=0\right)$. All three trials assessed interpersonal psychotherapy.

Meta-analysis with fixed-effect model on the data from the two trials $[69,80]$ reporting results on the BDI at follow-up were in agreement with the results from HDRS (mean difference on -5.54 BDI $\left(95 \%\right.$ CI -9.24 to $\left.-1.85 ; \mathrm{P}=0.001, \mathrm{I}^{2}=0\right)$.

Adverse events. Burnand et al. reported numbers of hospitalizations, days of hospitalization, and lost workdays in the different intervention groups [67]. They found significantly fewer 
Table 1. Characteristics of the included trials.

\begin{tabular}{|c|c|c|c|}
\hline Trials & Participants (randomized) & Interventions & Outcomes \& notes \\
\hline DiMascio et al., 1979 & 48 & $\begin{array}{l}\text { Interpersonal psychotherapy (individual } \\
16 \text { weeks) versus supportive } \\
\text { psychotherapy 'on demand' (up to one } \\
\text { monthly session) }\end{array}$ & Raskin Depression Scale, HDRS \\
\hline Elkin et al., 1989 & 125 & $\begin{array}{l}\text { Interpersonal psychotherapy (individual } \\
16-20 \text { weeks) versus pill-placebo and } \\
\text { clinical management (support, } \\
\text { encouragement and advice if necessary) }\end{array}$ & HDRS, BDI, remission HDRS $(<7)$ \\
\hline Schulberg et al., 1996 & 185 & $\begin{array}{l}\text { Interpersonal psychotherapy ( } 16 \text { weekly } \\
\text { individual sessions followed by } 4 \text { monthly } \\
\text { sessions) versus physicians usual care } \\
\text { (various procedures commonly used by } \\
\text { primary care physicians) }\end{array}$ & HDRS and remission HDRS $(<8)$ \\
\hline Burnand et al., 2002 & 90 & $\begin{array}{l}\text { Psychodynamic psychotherapy } \\
\text { (individual sessions for } 10 \text { weeks) and } \\
125 \mathrm{mg} \text { clomipramine versus supportive } \\
\text { care (individual sessions for } 10 \text { weeks) } \\
\text { and } 125 \mathrm{mg} \text { clomipramine }\end{array}$ & $\begin{array}{l}\text { HDRS, days of hospitalization, } \\
\text { hospitalizations, lost work days, and } \\
\text { treatment failure (major depressive } \\
\text { disorder at } 10 \text { weeks) }\end{array}$ \\
\hline Schramm et al., 2007 & 130 & $\begin{array}{l}\text { Interpersonal psychotherapy (individual } \\
\text { and group for } 5 \text { weeks) and } \\
\text { antidepressants (sertralin, amitriptyline) } \\
\text { versus clinical management ( } 3 \text { weekly } \\
\text { psychoeducative and supportive sessions } \\
\text { for } 5 \text { weeks) and antidepressants } \\
\text { (sertralin, amitrityline) }\end{array}$ & $\begin{array}{l}\text { HDRS, BDI remission }(H D R S<8) \\
\text { Participants were inpatients }\end{array}$ \\
\hline Swartz et al., 2008 & 65 & $\begin{array}{l}\text { Interpersonal psychotherapy MOMS } \\
\text { ( } 9 \text { individual sessions) versus treatment } \\
\text { as usual (given referrals to mental health } \\
\text { clinics and told to seek treatment) }\end{array}$ & $\begin{array}{l}\text { HDRS, BDI. IPT MOMS differs from } \\
\text { standardized IPT: shorter, brief behavioral } \\
\text { strategies, specific strategies to assist } \\
\text { mothers in managing psychiatrically ill } \\
\text { offspring }\end{array}$ \\
\hline
\end{tabular}

doi:10.1371/journal.pone.0019044.t001

of these events in the intervention group. Schramm et al. found a non-significant higher tendency for participants in the 'treatment as usual' group to be hospitalized after end of treatment [69]. Finally, DiMascio et al. included records on hospitalizations [56]. One participant in the experimental group and two in the control group were hospitalized.

None of the remaining trials reported on adverse events.
Quality of life. None of the included trials assessed the effect on quality of life of the participants.

\section{Secondary outcome measures}

Three trials $[69,75,79]$ reported the proportion of participants without remission as a dichotomous outcome measure. We had planned to define remission as a Hamilton score of less than 8,

Table 2. Risk of bias.

\begin{tabular}{|c|c|c|c|c|c|c|c|c|c|}
\hline & $\begin{array}{l}\text { Allocation } \\
\text { sequence } \\
\text { generation? }\end{array}$ & $\begin{array}{l}\text { Allocation } \\
\text { concealment? }\end{array}$ & $\begin{array}{l}\text { Intention to } \\
\text { treat analysis? }\end{array}$ & $\begin{array}{l}\text { Blinding of } \\
\text { outcome } \\
\text { assessors? }\end{array}$ & $\begin{array}{l}\text { Comparability } \\
\text { of drop-outs } \\
\text { in intervention } \\
\text { groups? }\end{array}$ & $\begin{array}{l}\text { Free of } \\
\text { selective } \\
\text { outcome } \\
\text { measure } \\
\text { reporting? }\end{array}$ & $\begin{array}{l}\text { Free of } \\
\text { economic } \\
\text { bias? }\end{array}$ & $\begin{array}{l}\text { Free of } \\
\text { academic } \\
\text { bias? }\end{array}$ & $\begin{array}{l}\text { Overall } \\
\text { bias } \\
\text { assessment }\end{array}$ \\
\hline $\begin{array}{l}\text { DiMascio et al., } \\
1979\end{array}$ & Unclear & Unclear & No & Yes & No & Unclear & Unclear & Unclear & $\begin{array}{l}\text { High risk of } \\
\text { bias }\end{array}$ \\
\hline Elkin et al., 1989 & Unclear & Unclear & No & Unclear & No & Yes & Yes & Unclear & $\begin{array}{l}\text { High risk of } \\
\text { bias }\end{array}$ \\
\hline $\begin{array}{l}\text { Schulberg et al., } \\
1996\end{array}$ & Unclear & Unclear & Yes & Yes & Unclear & Unclear & Unclear & Unclear & $\begin{array}{l}\text { High risk of } \\
\text { bias }\end{array}$ \\
\hline $\begin{array}{l}\text { Burnand et al., } \\
2002\end{array}$ & Unclear & Unclear & No & No & Yes & Unclear & Unclear & Unclear & $\begin{array}{l}\text { High risk of } \\
\text { bias }\end{array}$ \\
\hline $\begin{array}{l}\text { Schramm et al., } \\
2007\end{array}$ & Yes & Unclear & No & Yes & Unclear & Unclear & Yes & Unclear & $\begin{array}{l}\text { High risk of } \\
\text { bias }\end{array}$ \\
\hline $\begin{array}{l}\text { Swartz et al., } \\
2008\end{array}$ & Unclear & Unclear & No & Unclear & Unclear & Unclear & Yes & Unclear & $\begin{array}{l}\text { High risk of } \\
\text { bias }\end{array}$ \\
\hline
\end{tabular}




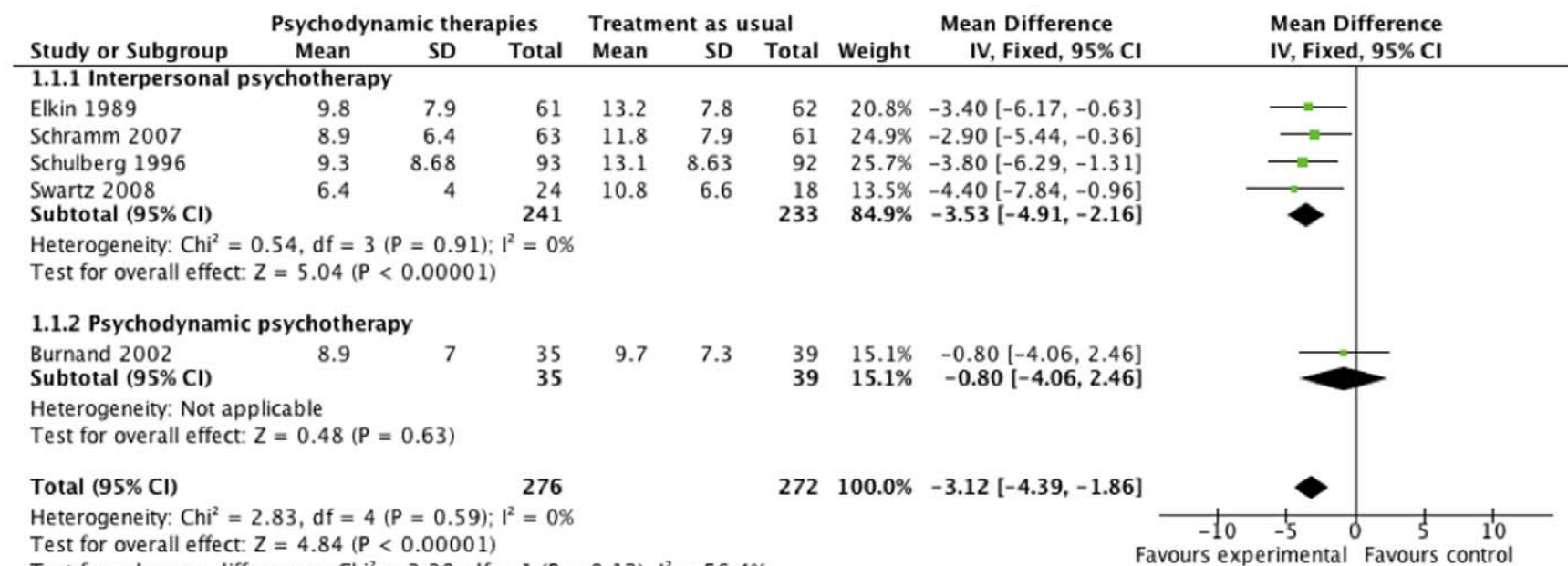

Figure 1. The effect of psychodynamic therapies at cessation of treatment on Hamilton Rating Scale for Depression. doi:10.1371/journal.pone.0019044.g001

TSMB variance $228.4 ;$ MIREDIF 2 ; a $5 \% ; \mathrm{b} 10 \%$ is a Two-sided graph

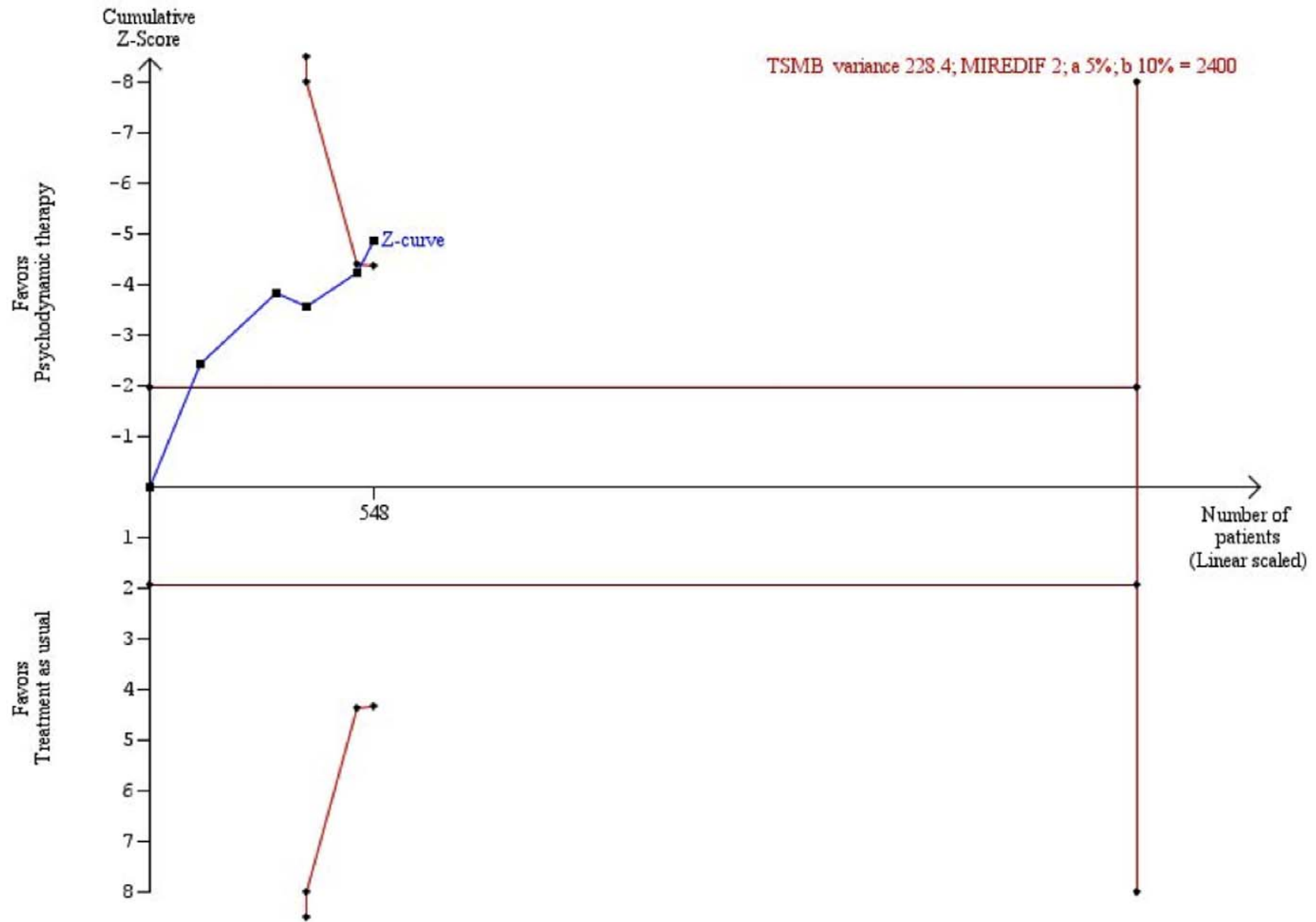

Figure 2. Trial sequential analysis of the cumulative meta-analysis of the effect of psychodynamic therapies versus 'treatment as usual' for major depressive disorder. The required information size of 2400 is calculated based on an intervention effect compared with 'treatment as usual', of 2 points on the HDRS, a variance of 228.4 on the mean difference, a risk of type I error of $5 \%$, and a power of $90 \%$. Even with these presumptions, the cumulated Z-curve (blue curve) crosses the trial sequential monitoring boundaries (red inner sloping lines) implying that there is firm evidence for a beneficial effect of psychodynamic therapies compared with 'treatment as usual'.

doi:10.1371/journal.pone.0019044.g002 
BDI less than 10, or MADRS less than 10. However, this was not possible, so we adopted the slightly different definitions of the individual trials. Two trials defined remission as HRDS less than 8 $[69,79]$. One trial defined remission in two different ways: HDRS less than 7 and BDI less than 10 [75]. In the latter trial the BDI data showed no significant difference in remission between the two intervention groups [75].

Meta-analysis on the HDRS-data from the three trials $[69,75,79]$ showed that psychodynamic therapy compared with 'treatment as usual' significantly decreases the risk of no remission with an odds ratio of 0.36 (95\% CI, 0.24 to 0.55 ; $\mathrm{P}=0.00001$, $\left.\mathrm{I}^{2}=2 \%\right)$. The number needed to treat to obtain one extra patient with remission is about four patients (95\% CI, 3 to 8). All three trials assessed interpersonal psychotherapy (Figure 3).

Only two of the trials $[56,69]$ included records of suicide attempts and suicides. Schramm et al. reported that one participant initially treated with 'standard care' committed suicide 10 days after cessation of treatment [69]. No other participants attempted suicide during the trial period. DiMascio et al. reported that none of the participants had suicide attempts or committed suicide during the trial period [56].

None of the trials reported on suicide inclination.

Random-effects model. None of our results were changed noticeably by conducting random-effects model meta-analysis.

Subgroup analyses. In subgroup analyses of therapists' level of education and experience (high versus intermediate versus unclear), type of therapy (group versus individual), and use of antidepressants as co-intervention (antidepressant co-intervention versus no antidepressant co-intervention), we found no heterogeneity in our results. This indicates that these factors do not seem to influence the effect of psychodynamic therapies.

We had also planned a subgroup-analysis according to risk of bias [22]. However, as all trials were classified as 'high risk of bias' it was not possible to conduct this analysis.

\section{Discussion}

The results of our systematic review with meta-analysis and trial sequential analysis show that randomized trials with low risk of systematic errors (bias) and low risk of random errors (play of chance) are needed. Psychodynamic therapies and especially interpersonal psychotherapy might significantly reduce depressive symptoms on the HDRS and increase the probability of remission compared with 'treatment as usual', but due to the high risk of systematic errors (bias) we cannot make any definite conclusions. The possible benefit measured on the HDRS is presumably small. The number needed to treat to obtain one extra patient with remission may be about four patients. The impact of psychodynamic therapies on suicidality, survival, and quality of life is unknown.
It could be argued that interpersonal psychotherapy is not a psychodynamic intervention. Interpersonal psychotherapy has its theoretical roots in psychodynamic therapy but has integrated elements from other therapies [14,15,84]. In spite of the integrative content of interpersonal psychotherapy we chose, as it's often done in the literature, to classify interpersonal psychotherapy as a form of psychodynamic therapy [14,85]. Furthermore, we believe that most forms of contemporary psychodynamic therapies in practice are delivered in a way similar to interpersonal psychotherapy.

\section{Strengths}

This review has a number of strengths. Our protocol was published before we began systematic literature searches in all relevant databases, data extraction, and data analyses. Data was extracted by two independent authors minimizing the risk of inaccurate data-extraction, and we assessed the risk of bias in all trials according to the Cochrane Handbook guidelines [20]. We meta-analyzed data both with fixed-effect and random-effects models and both analyses were in agreement in all our results. Furthermore, we performed trial sequential analysis to control for random error $[18,19,21]$. The results of the trial sequential analysis confirmed the cumulative meta-analysis result.

The characteristics of the participants in the different trials, as well as the severity of the depressive symptoms differed. E.g., one trial included only inpatients [69] and another trial included depressed mothers whose children were receiving psychiatric treatment [80]. Two of the trials used antidepressants as cointervention to psychodynamic therapies, and we included trials both assessing interpersonal psychotherapy and psychodynamic psychotherapy. Furthermore, the extent and form of the 'treatment as usual' condition varied greatly. We did not, however, find any heterogeneity in our analyses and found no difference on 'test of interaction' between interpersonal psychotherapy and psychodynamic psychotherapy. This indicates that there is a comparable treatment effect between interpersonal psychotherapy and other psychodynamic psychotherapies, between the different forms of 'treatment as usual', and among the different populations treated. This may make our results more generally applicable. On the other hand, few trials with few participants were included and only one trial used a psychodynamic intervention other than interpersonal psychotherapy. This decreases our power to detect any differences. Furthermore, in order to thoroughly examine a difference in effect between two interventions head-to-head comparisons are needed.

\section{Limitations}

Our systematic review has a number of limitations. Our results are based on only six trials with a limited number of participants. Also, all six trials had high risk of bias - so our results may be

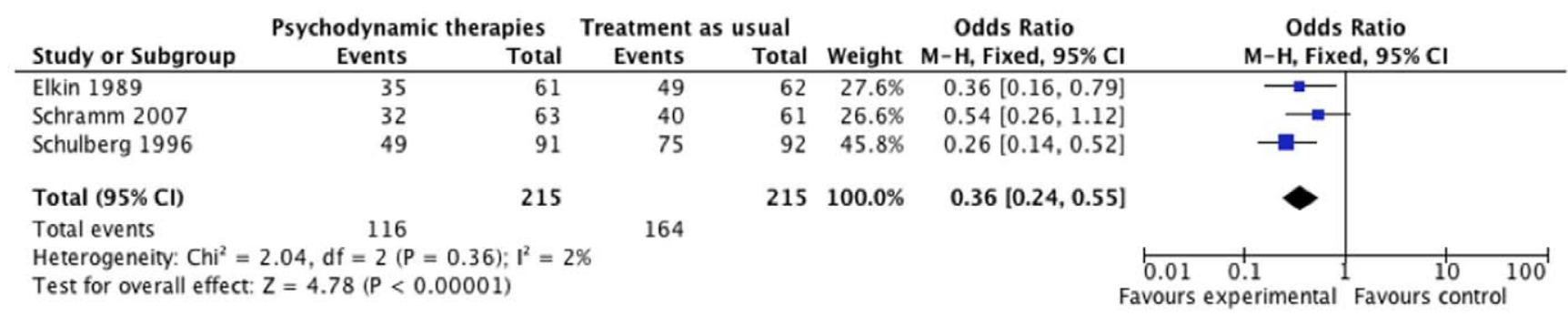

Figure 3. Effect of interpersonal psychotherapy on remission. Events: participants not remitting. doi:10.1371/journal.pone.0019044.g003 
questionable. Only three of the trials used an intervention that we classified as 'adequately defined', i.e., using and documenting the use of a therapeutic manual. In clinical trials it is imperative that the interventions are adequately defined and described [86]. Factors like personal style, communication skills, and personality of the therapist evidently will influence the way psychotherapy is delivered [87]. It is difficult to describe and control for these subjective factors, and this makes it even more important to relate the therapy to a treatment manual. Otherwise it is unclear what kind of intervention the participants were receiving, and it is difficult to apply any result in clinical practice. Moreover, a number of subgroups of depressed patients were not included in the trials of this review. These subgroups may react differently to psychotherapy and of course our review cannot be generalized to other than the included patient groups.

None of the trials reported measures of quality of life. Outcome measures of quality of life are generally not standardized and thoroughly individually validated [88]. The use of standardized outcome measures for quality of life in research has been limited by difficulties in administering and scoring quality of life [88], but quality of life can be used as a valid outcome measure [35,88].

Typically, adverse events are not reported as thoroughly as beneficial outcome measures [89], and only two of the included trials included records of numbers of suicides and suicide attempts, and only three trials reported on some adverse events. Some psychological interventions might have harmful effects. Psychological debriefing for preventing post-traumatic stress disorder is one example [90]. Debriefing has in some clinical trials showed to have a harmful effect [90]. Possible harmful effects of this kind of therapy are therefore not thoroughly examined.

\section{Implications}

Our results show that the possible benefit from this relatively extensive treatment compared with 'treatment as usual' was only a few points on the HDRS. From a clinical point of view it could be argued that this possible benefit is not clinically relevant especially if you relate this mean difference to the extent and length of the intervention. On the other hand, our analyses demonstrate that the number needed to treat to obtain one extra patient in remission was only about four patients. The latter estimate was based on only two trials, which primarily defined remission as Hamilton score under a given value.

The HDRS might not be a useful instrument to quantify the effect of psychodynamic therapies. Other assessment methods could demonstrate a more substantial effect of any given intervention for depression. Furthermore, severity of depression as measured by the total HDRS score has failed to predict suicide

\section{References}

1. Levav I, Rutz W (2002) The WHO world health report 2001. New understanding- new hope. Israel Journal of Psychiatry \& Related Sciences 39: 50-56.

2. Greenberg P, Stiglin LE, Finkelstein SN, Berndt ER (1993) The economic burden of depression in 1990. J Clin Psychiatry. pp 405-418.

3. Kessler RC, McGnagle KA, Zhao S, Nelson CB, Hughes M, et al. (1994) Lifetime and 12- month prevalence of DSM - III- R psychiatric disorders in the united states: Results from the Natinal Comorbidity Survey. Arch Gen Psychiatry 51: 8-19.

4. Spijker J, de GR, Bijl RV, Beekman AT, Ormel J, et al. (2002) Duration of major depressive episodes in the general population: results from The Netherlands Mental Health Survey and Incidence Study (NEMESIS). Br J Psychiatry 181: 208-213.

5. Arnow BA, Constantino MJ (2003) Effectiveness of psychotherapy and combination treatment for chronic depression. Journal of Clinical Psychology. pp 893-905.

6. Bech P (1999) Stress \& livskvalitet (Stress \& quality of life).

7. Fawcett $J(1993)$ The morbitity and mortality of clinical depression. International Clinical Psychopharmacology. pp 217-220. attempts [91], and some publications have questioned the usefulness of the HDRS and concluded that the scale is psychometrically and conceptually flawed [92]. The two other outcome measures often used to assess depressive symptoms, MADRS and BDI, probably correspond to HDRS [93,94]. The HDRS has during 40 years been the gold standard to quantify depressive symptoms in clinical trials [92]. There may be a need for other assessment methods.

A recently published meta-analysis examined the effect of shortterm psychodynamic psychotherapy for depression [16]. As mentioned in the above, the meta-analysis did not include thorough assessment of bias risk in the included trials, did not include trials using interpersonal psychotherapy as experimental intervention, and did not employ trial sequential analysis or other methods to reduce the risk of random errors [17-19]. However, the results showed a significant effect of short-term psychodynamic psychotherapy on depressive symptoms and this result supports the validity of our results in the present systematic review.

Future research should focus on comparing different forms of manualized psychotherapy - or comparing psychodynamic therapy with other treatments for depression. First and foremost such trials should be conducted with low risk of bias and low risk of random errors. Such trials should also report on adverse events, suicide inclination, suicide attempts, and numbers of suicides. There may be a need for a new gold standard assessment method other than HRDS to assess depressive symptoms, and if possible more effective interventions for depression must be developed.

\section{Conclusions}

Randomized trials with low risk of systematic errors (bias) and low risk of random errors (play of chance) are needed. Psychodynamic therapy, and especially interpersonal psychotherapy, might be an effective intervention for major depressive disorder compared with 'treatment as usual', but the possible treatment effect measured on the HDRS is small. The impact of psychodynamic therapies on suicidality, survival, and quality of life is unknown.

\section{Supporting Information}

Figure S1 PRISMA flowchart. (TIFF)

\section{Author Contributions}

Analyzed the data: JCJ JLH CG ES. Wrote the paper: JCJ CG.

8. Cipriani A, Santilli C, Furukawa TA, Signoretti A, Nakagawa A, et al. (2009) Escitalopram versus other antidepressive agents for depression. Cochrane Database Syst Rev CD006532., 10.1002/14651858.CD006532.pub2 [doi].

9. Kirsch I, Deacon BJ, Huedo-Medina TB, Scoboria A, Moore TJ, et al. (2008) Initial severity and antidepressant benefits: a meta-analysis of data submitted to the Food and Drug Administration. PLoS Medicine 5.

10. Turner EH, Matthews AM, Linardatos E, Tell RA, Rosenthal R (2008) Selective publication of antidepressant trials and its influence on apparent efficacy. N Engl J Med 358: 252-260. 358/3/252 [pii];10.1056/NEJMsa065779 [doi].

11. Geddes JR, Carney SM, Davies C, Furukawa TA, Kupfer DJ, et al. (2003) Relapse prevention with antidepressant drug treatment in depressive disorders: a systematic review. Lancet 361: 653-661. S0140-6736(03)12599-8 [pii];10.1016/ S0140-6736(03)12599-8 [doi].

12. Trede K (2007) 150 years of Freud-Kraepelin dualism. Psychiatr Q 78: 237-240. 10.1007/s11126-007-9036-0 [doi].

13. Kessing LV, Hansen HV, Hougaard E, Hvenegaard A, Albæk J (2006) Forebyggende ambulant behandling ved svær affektiv lidelse (depression og mani)- En medicinsk teknologi vurdering (preventive outpatient treatment for severe affective disorder). (Danish). Puljeprojekter; 6(9). 
14. Cornes C, Frank E (1994) Interpersonal psychotherapy for depression. The Clinical Psychologist 47: 9-10.

15. Weissman M, Markowitz J, Klerman G (2000) Comprehensive guide to interpersonal psychotherapy. Basic Books.

16. Driessen E, Cuijpers P, de Maat SCM, Abbass AA, de JF, et al. (2010) The efficacy of short-term psychodynamic psychotherapy for depression: A metaanalysis. Clinical Psychology Review 30: 25-36.

17. Brok J, Thorlund K, Gluud C, Wetterslev J (2008) Trial sequential analysis reveals insufficient information size and potentially false positive results in many meta-analysis. J Clin Epidemiol. pp 763-769.

18. Wetterslev J, Thorlund K, Brok J, Gluud C (2008) Trial sequential analysis may establish when firm evidence is reached in cumulative meta-analysis. J Clin Epidemiol 61: 64-75. S0895-4356(07)00147-3 [pii];10.1016/j.jclinepi.2007.03.013 [doi].

19. Thorlund K, Devereaux PJ, Wetterslev J, Guyatt G, Ioannidis JP, et al. (2009) Can trial sequential monitoring boundaries reduce spurious inferences from meta-analyses? Int J Epidemiol 38: 276-286. dyn179 [pii];10.1093/ije/dyn179 [doi].

20. Higgins J, Green S (2008) Cochrane Handbook for Systematic Reviews of interventios, Version 5.0.0.

21. Brok J, Thorlund K, Gluud C, Wetterslev J (2008) Trial sequential analysis reveals insufficient information size and potentially false positive results in many meta-analysis. J Clin Epidemiol. pp 763-769.

22. Jakobsen J, Lindschou Hansen J, Simonsen E, Gluud C (2010) The effect of psychodynamic therapy versus treatment as usual in patients with major depressive disorder. A systematic review of randomised clinical trials with metaanalyses and trial sequential analyses (protocol). Published at www ctu dk.

23. American Psychiatric Association (1994) Diagnostic and Statistical Manual of Mental Disorders.

24. World Health Organization (1992) International Statistical Classification of Diseases an Related Health Problems (10th Revision) ICD 10.

25. American Psychiatric Association (1980) Diagnostic and Statistical Manual of Mental Disorders (DSM III).

26. American Psychiatric Association (1987) Diagnostic and Statistical Manual of Mental Disorders (DSM III-R).

27. Sofuoglu M, Sugarman DE, Carroll KM (2010) Cognitive function as an emerging treatment target for marijuana addiction. Exp Clin Psychopharmacol 18: 109-119. 2010-06751-001 [pii];10.1037/a0019295 [doi].

28. Davidson KW, Rieckmann N, Clemow L, Schwartz JE, Shimbo D, et al. (2010) Enhanced depression care for patients with acute coronary syndrome and persistent depressive symptoms: coronary psychosocial evaluation studies randomized controlled trial. Arch Intern Med 170: 600-608. 170/7/600 [pii];10.1001/archinternmed.2010.29 [doi].

29. Wilkins CH, Mathews J, Sheline YI (2009) Late life depression with cognitive impairment: evaluation and treatment. Clin Interv Aging 4: 51-57.

30. Howard M, Battle GL, Pearlstein T, Rosene-Montella K (2006) A psychiatric mother-baby day hospital for pregnant and postpartum women. Arch Womens Ment Health 9: 213-218. 10.1007/s00737-006-0135-y [doi].

31. Derksen JJ (2006) [Short-term psychodynamic psychotherapy]. Tijdschr Psychiatr 48: 777-786.

32. Hoglend P, Amlo S, Marble A, Bogwald K-P, Sorbye O, et al. (2006) Analysis of the patient-therapist relationship in dynamic psychotherapy: An experimental study of transference interpretations. Am J Psychiatry 163: 1739-1746.

33. Gluud LL (2006) Bias in clinical intervention research. Am J Epidemiol. pp 493-501.

34. Wood L, Egger M, Gluud LL, Schulz KF, Juni P, et al. (2008) Empirical evidence of bias in treatment effect estimates in controlled trials with different interventions and outcomes: meta-epidemiological study. BMJ 336: 601-605. bmj.39465.451748.AD [pii];10.1136/bmj.39465.451748.AD [doi].

35. Gluud C (2006) The culture of designing hepato-biliary randomised trials. J Hepatol 44: 607-615. S0168-8278(05)00823-8 [pii];10.1016/ j.jhep.2005.12.006 [doi].

36. Kjaergaard L, Villumsen J, Gluud C (2001) Reported methodologic quality and discrepancies between large and small randomized trials in meta-analysis. ANN INTERN MED 135: 982-989.

37. Hamilton M (1960) A rating scale for depression. J NEUROL NEUROSURG PSYCHIATRY 23: 56-61.

38. Bech AT (1961) An inventory for measuring depression. Archives of General Psychiatry. pp 561-571.

39. Montgomery SA, Asberg M (1979) A new depression scale designed to be sensitive to change. Br J Psychiatry 134: 382-389.

40. ICH-GCP (1997) Code of Federal Regulations \& Guidelines Vol. 1. International Committee on Harmonization Philadelphia, US: Barnett International/PAREXEL, 1997.

41. The Nordic Cochrane Centre TCG (2008) Review Manager (RevMan) [Computer program]. Version 5.0. Copenhagen: The Nordic Cochrane Centre, The Cochrane Collaboratio.

42. Bass J, Neugebauer R, Clougherty KF, Verdeli H, Wickramaratne P, et al. (2006) Group interpersonal psychotherapy for depression in rural Uganda: 6month outcomes: randomised controlled trial. Br J Psychiatry 188: 567-573.

43. Lang AJ, Norman GJ, Casmar PV (2006) A randomized trial of a brief mental health intervention for primary care patients. Journal of Consulting \& Clinical Psychology 74: 1173-1179.
44. Miller L, Weissman M (2002) Interpersonal psychotherapy delivered over the telephone to recurrent depressives: A pilot study. Depression and Anxiety 16: 117.

45. Simpson S, Corney R, Fitzgerald P, Beecham J (2000) A randomised controlled trial to evaluate the effectiveness and cost-effectiveness of counselling patients with chronic depression. Health Technology Assessment 4: i+iii-74.

46. Rodriguez JL, Butron MAL, Terrez BEV, Salcedo VV (2004) Double blind study with antidepressant, brief psychotherapy and placebo in patients with mild to moderate depression (Spanish). Salud Mental 27: 55-61.

47. Coulehan JL, Schulberg HC, Block MR, Madonia MJ, Rodriguez E (1997) Treating depressed primary care patients improves their physical, mental, and social functioning. Arch Intern Medicine 157: 1113-1120.

48. Vitriol VG, Ballesteros ST, Florenzano RU, Weil KP, Benadof DF (2009) Evaluation of an outpatient intervention for women with severe depression and a history of childhood trauma. Psychiatric services 60: 936-942.

49. de Arufo CB, Torres-Torija C, Biagini Alarcon M, Munoz MdCL (2000) Effects of group psychotherapy in outpatients. (Spanish). Psiquiatria 16: -56 .

50. Bolton P, Bass J, Neugebauer R, Verdeli H, Clougherty KF, et al. (2003) Group interpersonal psychotherapy for depression in rural Uganda: a randomized controlled trial. JAMA 289: 3117-3124.

51. Levkovitz Y, Shahar G, Native G, Hirsfeld E, Treves I, et al. (2000) Group interpersonal psychotherapy for patients with major depression disorder - pilot study. Journal of Affective Disorders 60: 191-195.

52. Maina G, Forner F, Bogetto F (2005) Randomized controlled trial comparing brief dynamic and supportive therapy with waiting list condition in minor depressive disorders. Psychotherapy and psychosomatics 74: 43-50.

53. Piper WE, Azim HF, McCallum M, Joyce AS (1990) Patient suitability and outcome in short-term individual psychotherapy. Journal of Consulting \& Clinical Psychology 58: 475-481.

54. Simpson S, Corney R, Beecham J (2003) A randomized controlled trial to evaluate the effectiveness and cost-effectiveness of psychodynamic counselling for general practice patients with chronic depression. Psychological Medicine 33: 229-239.

55. De Jonghe F, Kool S, van AG, Dekker J, Peen J (2001) Combining psychotherapy and antidepressants in the treatment of depression. J Affect Disord 64: 217-229.

56. DiMascio A, Weissman MM, Prusoff BA, Neu C, Zwilling M, et al. (1979) Differential symptom reduction by drugs and psychotherapy in acute depression. Arch Gen Psychiatry 36: 1450-1456.

57. Weissman MM, Prusoff BA, DiMascio A, Neu C, Goklaney M, et al. (1979) The efficacy of drugs and psychotherapy in the treatment of acute depressive episodes. Am J Psychiatry 136: 555-558.

58. Weissman MM, Klerman GL, Prusoff BA, Sholomskas D, Padian N (1981) Depressed outpatients. Results one year after treatment with drugs and/or interpersonal psychotherapy. Arch Gen Psychiatry 38: 51-55.

59. Ye HP, Ming L (2006) Group Interpersonal Psychotherapy for Inpatient with Major Depression. [Chinese]. Chinese Mental Health Journal 20: -526.

60. Kool S, Dekker J, Duijsens IJ, de JF, Puite B (2003) Efficacy of combined therapy and pharmacotherapy for depressed patients with or without personality disorders. Harvard Review of Psychiatry 11: 133-141.

61. Kool S, Dekker J, Duijsens IJ, de JF, Puite B (2003) Changes in personality pathology after pharmacotherapy and combined therapy for depressed patients. Journal of Personality Disorders 17: 60-72.

62. Kool S, Schoevers R, Duijsens IJ, Peen J, van AG, et al. (2007) [Treatment of depressive disorder and comorbid personality pathology: combined therapy versus pharmacotherapy]. [Dutch]. Tijdschrift voor Psychiatrie 49(6): 361-72.

63. Molenaar PJ, Dekker J, Van R, Hendriksen M, Vink A, et al. (2007) Does adding psychotherapy to pharmacotherapy improve social functioning in the treatment of outpatient depression? Depression \& Anxiety 24: 553-562.

64. Bellino S, Zizza M, Rinaldi C, Bogetto F (2006) Combined treatment of major depression in patients with borderline personality disorder: a comparison with pharmacotherapy. Canadian Journal of Psychiatry 51: 453-460.

65. Blom MB, Jonker K, Dusseldorp E, Spinhoven P, Hoencamp E, et al. (2007) Combination treatment for acute depression is superior only when psychotherapy is added to medication. Psychotherapy and Psychosomatics 76: 289-297.

66. Blom MB, Spinhoven P, Hoffman T, Jonker K, Hoencamp E, et al. (2007) Severity and duration of depression, not personality factors, predict short term outcome in the treatment of major depression. J Affect Disord 104: 119-126.

67. Burnand Y, Andreoli A, Kolatte E, Venturini A, Rosset N (2002) Psychodynamic psychotherapy and clomipramine in the treatment of major depression. PSYCHIATR SERV 53: -590.

68. Shea MT, Elkin I, Imber SD, Sotsky SM (1992) Course of depressive symptoms over follow-up: Findings from the National Institute of Mental Health Treatment of Depression Collaborative Research Program. Archives of General Psychiatry 49: 782-787.

69. Schramm E, van CD, Dykierek P, Lieb K, Kech S, et al. (2007) An intensive treatment program of interpersonal psychotherapy plus pharmacotherapy for depressed inpatients: acute and long-term results. Am J Psychiatry 164: 768-777.

70. Kech S, Zobel I, Dykierek P, van Calker D, Berger M, et al. (2008) Interpersonal psychotherapy for inpatients with depression. Effects on social adjustment and interpersonal problems. [German]. Zeitschrift fur Klinische Psychologie und Psychotherapie: Forschung und Praxis 37: 81-88. 
71. van CD, Zobel I, Dykierek P, Deimel CM, Kech S, et al. (2009) Time course of response to antidepressants: predictive value of early improvement and effect of additional psychotherapy. Journal of Affective Disorders 114: 243-253.

72. Imber SD, Pilkonis PA, Sotsky SM, Elkin I, Watkins JT, et al. (1990) Modespecific effects among three treatments for depression. Journal of Consulting and Clinical Psychology 58: 352-359.

73. Sotsky SM, Glass DR, Shea MT, Pilkonis PA (1991) Patient predictors of response to psychotherapy and pharmacotherapy: Findings in the NIMH Treatment of Depression Collaborative Research Program. Am J Psychiatry 148: 997-1008.

74. Stewart JW, Garfinkel R, Nunes EV, Donovan S, Klein DF (1998) Atypical features and treatment response in the National Institute of Mental Health Treatment of Depression Collaborative Research Program. Journal of clinical psychopharmacology 18: 429-434

75. Elkin I, Shea MT, Watkins JT, Imber SD, Sotsky SM, et al. (1989) National Institute of Mental Health Treatment of Depression Collaborative Research Program. General effectiveness of treatments. Arch Gen Psychiatry 46: 971-982.

76. Swartz HA, Zuckoff A, Frank E, Spielvogle HN, Shear MK, et al. (2006) An open-label trial of enhanced brief interpersonal psychotherapy in depressed mothers whose children are receiving psychiatric treatment. Depression and Anxiety 23: 398-404.

77. Elkin I, Parloff MB, Hadley SW, Autry JH (1985) NIMH treatment of Depression Collaborative Research Program: Background and research plan. Arch Gen Psychiatry 42: 305-316.

78. Schramm E, Schneider D, Zobel I, van CD, Dykierek P, et al. (2008) Efficacy of Interpersonal Psychotherapy plus pharmacotherapy in chronically depressed inpatients. Journal of Affective Disorders 109: 65-73.

79. Schulberg HC, Block MR, Madonia MJ, Scott CP, Rodriguez E, et al. (1996) Treating major depression in primary care practice. Eight-month clinical outcomes. Arch Gen Psychiatry 53: 913-919.

80. Swartz HA, Frank E, Zuckoff A, Cyranowski JM, Houck PR, Cheng Y, et al. (2008) Brief interpersonal psychotherapy for depressed mothers whose children are receiving psychiatric treatment. Am J Psychiatry 165: 1155-1162.

81. Raskin A, Schulterbrandt J, Reatig N, McKeon JJ (1969) Replication of factors of psychopathology in interview, ward behavior and self-report ratings of hospitalized depressives. Journal of Nervous \& Mental Disease 148: 87-98.
82. Burnand Y, Andreoli A, Kolatte E, Venturini A, Rosset N (2002) Psychodynamic psychotherapy and clomipramine in the treatment of major depression. PSYCHIATR SERV 53: 585-590.

83. Altman DG, Bland JM (2003) Interaction revisited: the difference between two estimates. BMJ 326: 219.

84. Cutler JL, Goldyne A, Markowitz JC, Devlin MJ, Glick RA (2004) Comparing cognitive behavior therapy, interpersonal psychotherapy, and psychodynamic psychotherapy. Am J Psychiatry 161: 1567-1573.

85. Levenson H, Butler SF, Powers TA, Beitman BD (2002) Concise guide to Brief Dynamic and Interpersonal Therapy. American Psychiatric Publishing Inc.

86. Boutron I, Moher D, Altman DG, Schulz KF, Ravaud P (2008) Extending the CONSORT statement to randomized trials of nonpharmacologic treatment: explanation and elaboration. Ann Intern Med 148: 295-309. 148/4/295 [pii].

87. Walwyn R, Roberts C (2010) Therapist variation within randomised trials of psychotherapy: implications for precision, internal and external validity. Stat Methods Med Res 19: 291-315. 0962280209105017 [pii];10.1177/ 0962280209105017 [doi].

88. Higginson IJ, Carr AJ (2001) Measuring quality of life: Using quality of life measures in the clinical setting. BMJ 322: 1297-1300.

89. Hopewell S, Wolfenden L, Clarke M (2008) Reporting of adverse events in systematic reviews can be improved: survey results. J Clin Epidemiol 61: 597-602. S0895-4356(07)00367-8 [pii];10.1016/j.jclinepi.2007.10.005 [doi]

90. Rose S, Bisson J, Churchill R, Wessely S (2002) Psychological debriefing for preventing post traumatic stress disorder (PTSD).

91. Chakraborty R, Chatterjee A (2007) Predictors of Suicide Attempt Among those with Depression in an Indian Sample: A Brief Report. The Internet Journal of Mental Health 4.

92. Bagby RM, Ryder AG, Schuller DR, Marshall MB (2004) The Hamilton Depression Rating Scale: has the gold standard become a lead weight? Am J Psychiatry 161: 2163-2177. 161/12/2163 [pii];10.1176/appi.ajp.161.12.2163 [doi].

93. Fitzgibbon ML, Cella DF, Sweeney JA (1988) Redundancy in measures of depression. J Clin Psychol 44: 372-374.

94. Heo M, Murphy CF, Meyers BS (2007) Relationship between the Hamilton Depression Rating Scale and the Montgomery-Asberg Depression Rating Scale in depressed elderly: a meta-analysis. Am J Geriatr Psychiatry 15: 899-905. 15/ 10/899 [pii]. 